Pacific Journal of Mathematic 


\title{
DIFFERENTIAL EXTENSION FIELDS OF EXPONENTIAL TYPE
}

\author{
MAXWELL RosenLICHT
}

\begin{abstract}
The special properties of differential extension fields which can be generated by elements with logarithmic derivatives in the base field are worked out. The results are analogous to those for Kummer extensions of ordinary fields, where $n^{\text {th }}$ roots are adjoined. The problem of the integration in finite terms of elements of such extension fields is considered, with applications to certain distribution functions that occur in statistics.
\end{abstract}

1. By a differential field is here meant a field $k$ together with an indexed family $\left\{D_{i}\right\}_{i \in I}$ of derivations of $k$. For brevity, we speak of "the differential field $k$ ", referring to the whole combination, and of "the given derivations of $k$ ", referring to the set $\left\{D_{i}\right\}_{i \in I}$. The constants of the differential field $k$ are $\bigcap_{i \in I} \operatorname{ker} D_{i}$, a subfield of $k$. A differential extension field of $k$ is an extension field $K$ of $k$ together with a family of derivations $\left\{D_{i}^{\prime}\right\}_{i \in I}$ of $K$ indexed by the same set such that the restriction of each $D_{i}^{\prime}$ to $k$ is $D_{i}$.

If $k$ is a differential field and $x$ a nonzero element of some differential extension field $K$ of $k$, we say that $x$ is exponential over $k$ if $D x / x \in k$ for each given derivation $D$ of $K$; in virtue of the "logarithmic derivative identity" $D x / x+D y / y=D(x y) /(x y)$, the set of all elements of $K$ that are exponential over $k$ forms a multiplicative subgroup of $K$ that contains the multiplicative group $k^{*}$ of $k$.

Part of the following result occurs in [2, p. 1156].

THEOREM 1. Let $k$ be a differential field of characteristic zero, $K$ a differential extension field of $k$ with the same subfield of constants. Any element of $K$ which can be written as a finite sum $\sum_{i} y_{i}$, where each $y_{i}$ is an element of $K$ that is exponential over $k$ and $y_{i} / y_{j} \notin k$ if $i \neq j$, can be written as such a sum in only one way; in this case $\sum_{i} y_{i}$ is algebraic over $k$ if and only if each $y_{i}$ is algebraic over $k$, which is true if and only if some power of $y_{i}$ is in $k$. If $K=k\left(x_{1}, \cdots, x_{n}\right)$, with each $x_{i}$ exponential over $k$, then the multiplicative group $E$ of all elements of $K$ which are exponential over $k$ is generated by $x_{1}, \cdots, x_{n}$ and $k^{*}$, and the abelian group $E / k^{*}$ has rank deg.tr. $K / k$ and torsion subgroup of order [(algebraic closure of $k$ in $K): k]$.

If the first statement is false, we can find elements $y_{1}, \cdots, y_{N}$ of $K$ that are exponential over $k$, with $y_{i} / y_{j} \notin k$ if $i \neq j$, such that 
$\sum_{i=1}^{N} y_{i}=0$. Suppose that such $y_{1}, \cdots, y_{N}$ are chosen with $N$ minimal. Then $N>1$ and for any given derivation $D$ of $K$ we have $\sum_{i=1}^{N} D y_{i}=0$, so that $\sum_{i=1}^{N}\left(D y_{i} / y_{i}-D y_{1} / y_{1}\right) y_{i}=0$, which is a relation of the unwanted type with effectively smaller $N$ unless for each $i=1, \cdots, N$ we have $D y_{i} / y_{i}=D y_{1} / y_{1}$, or $D\left(y_{i} / y_{1}\right)=0$. Since this is true for each given derivation $D, y_{i} / y_{1}$ is a constant, hence an element of $k$, a contradiction. Therefore our first contention is true. Note that this implies that if $y_{1}, \cdots, y_{N} \in K$ are exponential over $k$ and $y_{i} / y_{j} \notin k$ if $i \neq j$ then $\sum_{i=1}^{N} y_{i}$ is exponential over $k$ if and only if $N=1$, and also that any polynomial relation with coefficients in $k$ that is satisfied by $y_{1}, \cdots, y_{N}$ is a sum of binomial relations, each got from an equality of monomials. This last statement implies that any $y \in K$ that is exponential over $k$ and algebraic over $k$ has some power in $k$; conversely, the equality $D y^{m} / y^{m}=m D y / y$ shows that if a power of $y$ is in $k$ then $y$ is exponential over $k$. We now claim that if $y_{1}, \cdots$, $y_{N} \in K$ are exponential over $k$, with $y_{i} / y_{j} \notin k$ if $i \neq j$, and $\sum_{i=1}^{N} y_{i}$ is algebraic over $k$, then each $y_{i}$ is algebraic over $k$. For if not, take such $y_{1}, \cdots, y_{N}$ that give a counterexample with minimal $N$. Then $N>1$ and for any given derivation $D$ of $K$ we have $\sum_{i=1}^{N} D y_{i}$ algebraic over $k$, hence also $\sum_{i=1}^{N}\left(D y_{i} / y_{i}-D y_{1} / y_{1}\right) y_{i}$ is algebraic over $k$. Since the last expression has at most $N-1$ nonzero terms, all $D y_{i} / y_{i}-$ $D y_{1} / y_{1}$ must be zero, implying as before that each $y_{i} / y_{1} \in k$, a contradiction. All the rest of what we wish to show, except for the statement on the order of the torsion subgroup of $E / k^{*}$, will now be proved by induction on $n$. The case $n=0$ is trivial. If $n>1$ and our statements are true for $n-1$, then applying them to the fields $k \subset k\left(x_{1}, \cdots, x_{n-1}\right)$ reduces us immediately to the case $n=1$. Therefore suppose $K=k(x)$, with $x$ exponential over $k$. If $x$ is algebraic over $k$ and we set $[K: k]=m$, then any element of $K$ can be uniquely written in the form $\sum_{i=0}^{m-1} a_{i} x^{i}$ with each $a_{i} \in k$, and what we have already shown indicates that only elements of the form $a_{i} x^{i}$ are exponential over $k$, proving that $E$ is generated by $x$ and $k^{*}$; in this case we also note that both the rank and transcendence degree are zero and that the statement about the order of the torsion subgroup of $E / k^{*}$ is verified. If $K=k(x)$, where $x$ is both exponential over $k$ and transcendental over $k$, then any nonzero element of $K$ can be written as $f / g$, where $f$ and $g$ are relatively prime elements of $k[x]$. To prove that in this case too $E$ is generated by $x$ and $k^{*}$, it suffices to show that if $f$ and $g$ are relatively prime elements of $k[x]$ with constant terms 1 and $f / g$ is exponential over $k$ then $f=$ $g=1$. To do this, note that for any given derivation $D$ of $K$ we have $D f, D g$ elements of $k[x]$ of degrees at most those of $f, g$ respectively and with zero constant terms, and since $f / g$ is exponential over $k$ we have $D f / f-D g / g \in k$, so that $f g$ divides $g D f-f D g$, so 
that $f$ and $g$ divide $D f$ and $D g$ respectively, implying $D f=D g=0$; since this is true for all $D, f$ and $g$ are constants, hence elements of $k$, hence equal to 1 . In this case $\operatorname{rank} E / k^{*}=1=\operatorname{deg} . \operatorname{tr} . E / k$. It therefore remains only to prove the part about the torsion subgroup. For this, we use the fundamental theorem on abelian groups to replace $x_{1}, \cdots, x_{n}$, if necessary, by other elements $x_{1}, \cdots, x_{n}$, such that $x_{1} k^{*}, \cdots, x_{r} k^{*}$ generate the torsion subgroup of $E / k^{*}$ while $x_{r+1} k^{*}, \cdots, x_{n} k^{*}$ are a minimal set of generators for a complementary free subgroup. Here deg. tr. $K / k=\operatorname{rank} E / k^{*}=n-r$, so that $x_{r+1}$, $\cdots, x_{n}$ are algebraically independent over $k$, and therefore the algebraic closure of $k$ in $K$ is $k\left(x_{1}, \cdots, x_{r}\right)$. We are therefore reduced to proving the contention about the torsion subgroup of $E / k^{*}$ in the case where $K$ is algebraic over $k$. In this case the above method of induction on $n$ works perfectly well, immediately reducing us to the case $n=1$, which was proved above. Note that once the finite generation of the group $E / k^{*}$ was proved, we could have altered $x_{1}, \cdots, x_{n}$, if necessary, to obtain $x_{1} k^{*}, \cdots, x_{r} k^{*}$ a minimal set of generators for the torsion subgroup of $E / k^{*}$ and $x_{r+1} k^{*}, \cdots, x_{n} k^{*}$ free generators for a complementary subgroup, and then both the rank and torsion subgroup statements would have followed directly.

2. If $x$ and $y$ are elements of a differential field $k$, with $y \neq 0$, then $x$ is called a logarithm of $y$, and $y$ an exponential of $x$, if $D x=D y / y$ for each given derivation $D$ of $k$. A differential extension field of $k$ is called an elementary extension of $k$ if it is of the form $k\left(t_{1}, \cdots, t_{N}\right)$, where for each $i=1, \cdots, N, t_{i}$ is either a logarithm or an exponential of an element of $k\left(t_{1}, \cdots, t_{i-1}\right)$, or algebraic over the latter field. Note that if $t_{1}, \cdots, t_{N}$ are constants then $k\left(t_{1}, \cdots, t_{N}\right)$ is an elementary extension of $k$. We quote from [6] the basic general theorem on the elementary integrability of functions.

LIOUVILLE'S THEOREM. Let $k$ be a differential field of characteristic zero and for each given derivation $D$ of $k$ let $\alpha_{D} \in k$. Then there exists an elementary differential extension field of $k$ having the same constants and containing an element $y$ such that $D y=\alpha_{D}$ for each given derivation $D$ if and only if there are constants $c_{1}, \cdots, c_{n} \in k$ and elements $u_{1}, \cdots, u_{n}, v \in k$ such that for each given derivation $D$ we have

$$
\alpha_{D}=\sum_{i=1}^{n} c_{i} \frac{D u_{i}}{u_{i}}+D v
$$

For completeness we include the proofs of the following lemma and proposition, which appear in somewhat less generality in [3, 
p. 87] and [4, p. 171] respectively.

LEmma. Let $k$ be a differential field, $K a$ differential extension field of $k$. Then $k$ and the constants of $K$ are linearly disjoint over the constants of $k$.

For if not, we can find constants $c_{1}, \cdots, c_{n}$ of $K$ that are linearly independent over the constants of $k$ but such that there exist $x_{1}, \cdots, x_{n} \in k$, not all zero, such that $c_{1} x_{1}+\cdots+c_{n} x_{n}=0$. Choose such $c_{1}, \cdots, c_{n}, x_{1}, \cdots, x_{n}$ with $n$ minimal and with $x_{1}=1$. For any given derivation $D$ of $K$, the equation $\sum_{i=2}^{n} c_{i} D x_{i}=0$ contradicts the minimality of $n$ unless each $D x_{i}=0$, from which it follows that each $x_{i}$ is a constant of $k$, contradicting the linear independence of $c_{1}, \cdots, c_{n}$ over the constants of $k$.

Proposition. Let $k$ be a differential field of characteristic zero and for each given derivation $D$ of $k$ let $\alpha_{D} \in k$. If there exists an elementary differential extension field of $k$ which contains an element $y$ such that $D y=\alpha_{D}$ for each given derivation $D$, then there exists such an extension field of $k$ whose subfield of constants is an algebraic extension of the subfield of constants of $k$.

For suppose such a $y$ exists in the elementary differential extension field $K$ of $k$. We may assume that $K$ is algebraically closed. Let $\mathscr{C}$ be the subfield of constants of $K$. Applying Liouville's theorem to the differential fields $\mathscr{C}(k)$ and $K$, we get elements $c_{1}, \cdots, c_{n} \in \mathscr{C}$ and $u_{1}, \cdots, u_{n}, v \in \mathscr{C}(k)$ such that for each given derivation $D$ of $K$ we have $\alpha_{D}=\sum_{i=1}^{n} c_{i} D u_{i} / u_{i}+D v$. Each element of $\mathscr{C}(k)$ is the quotient of sums of products of elements of $\mathscr{C}$ by elements of $k$. Using the logarithmic derivative identity and enlarging $n$ if necessary, we can get each $u_{i}$ to be such a sum of products. Hence we can write $u_{i}=\sum_{j=1}^{N} c_{i j} x_{j}$ for $i=1, \cdots, n$ and $v=\left(\sum_{j=1}^{N} a_{j} x_{j}\right) /\left(\sum_{j=1}^{N} b_{j} x_{j}\right)$, where each $c_{i j}, a_{j}, b_{j} \in \mathscr{C}$ and each $x_{j} \in k$. We can change $x_{1}, \cdots, x_{N}$ if necessary to be able to assume that they are linearly independent over the constant subfield $\mathscr{C}_{k}=\mathscr{C} \cap k$ of $k$. Choose integers $j(0), j(1), \cdots, j(n)$ from among $\{1,2, \cdots, N\}$ such that $b_{j(0)} c_{1 j(1)} \cdots c_{n j(n)} \neq 0$. Now consider undetermined constants $\left\{C_{i}, C_{i j}, A_{j}, B_{j}\right\}_{i=1, \cdots, n ; j=1, \cdots, N}$ of $\mathscr{C}$ such that $B_{j(0)} C_{1 j(1)} \cdots C_{n j(n)} \neq 0$ and impose the further condition that

$$
\alpha_{D}=\sum_{i=1}^{n} C_{i} \frac{D\left(\sum_{j=1}^{N} C_{i j} x_{j}\right)}{\sum_{j=1}^{N} C_{i j} x_{j}}+D\left(\frac{\sum_{j=1}^{N} A_{j} x_{j}}{\sum_{j=1}^{N} B_{j} x_{j}}\right)
$$


for each given derivation $D$ of $K$. Since $B_{j(0)} C_{1 j(1)} \cdots C_{n j(n)} \neq 0$, the expression for $\alpha_{D}$ is well-defined and the last equation can be cleared of fractions to get an equivalent equality with zero of a certain sum of products of elements of $\mathscr{C}_{k}\left[\left\{C_{i}, C_{i j}, A_{j}, B_{j}\right\}\right]$ by elements of $k$. The elements of $k$ appearing here can be taken to be linearly independent over $\mathscr{C}_{k}$, in which case the lemma implies that all the coefficients must be zero. Thus all the equations for all the $\alpha_{D}$ 's are equivalent to the annulling of a certain subset of $\mathscr{C}_{k}\left[\left\{C_{i}, C_{i j}, A_{j}, B_{j}\right\}\right]$. To get special values of $\left\{C_{i}, C_{i j}, A_{j}, B_{j}\right\}$ in the algebraic closure of $\mathscr{C}_{k}$ (a subfield of $K$ ) for which all the equations for $\alpha_{D}$ hold, and so to prove the proposition, we need only take a $\mathscr{C}_{k}$-specialization into the algebraic closure of $\mathscr{C}_{k}$ of $\left\{c_{i}, c_{i j}, a_{j}, b_{j}, 1 / b_{j(0)} c_{1 j(1)} \cdots c_{n j(n)}\right\}$.

For simplicity, the following result is stated and proved only for the case of ordinary differential fields, that is the case where there is only one given derivation $D$. The modifications necessary for the more general case are indicated later.

THEOREM 2. Let $k \subset k\left(x_{1}, \cdots, x_{n}\right)$ be ordinary differential fields of characteristic zero with the same subfield of constants and with each $x_{i}$ exponential over $k$ and suppose that $k$ contains a primitive $m$ th root of unity, where $m$ is the annihilator of the torsion subgroup of the group of elements of $k(x)$ that are exponential over $k$ modulo the multiplicative group of $k$, that is $m$ is the least positive integer such that if some power of an element of $k(x)$ is in $k$ then so is its $m$ th power. If $y_{1}, \cdots, y_{N} \in k(x)$ are exponential over $k$ and $y_{i} / y_{j} \notin k$ for $i \neq j$, then $y_{1}+y_{2}+\cdots+y_{N}$ is the derivative of an element in some elementary differential extension field of $k(x)$ having the same constant subfield if and only if each $y_{i}$ has this property; in this case, if $y_{i}$ is not algebraic over $k$ then it is the derivative of a $y_{i}$, for some $a \in k$, while if $y_{i}$ is algebraic over $k$ then it differs from an element of the form $\sum_{i=1}^{n} c_{i} x_{i}^{\prime} / x_{i}$, where each $c_{i}$ is a constant of $k$, by the derivative of an element of some elementary extension field of $k$ having the same constants.

If $y_{1}, \cdots, y_{N}$ are as given and each $y_{i}$ is the derivative of an element of some elementary extension field of $k(x)$ having the same constants, then each $y_{i}$ is of the form indicated in the statement of Liouville's theorem, with all relevant quantities in $k(x)$, hence $y_{1}+$ $\cdots+y_{N}$ is also of this form, so that the latter is the derivative of an element of some elementary extension field of $k(x)$ having the same constants. For the converse and main part of the theorem, we first consider the special case where $x_{1}, \cdots, x_{n}$ are algebraically independent over $k$, a case where $m=1$. Each $y_{i}$ here can be written uniquely as the product of an element of $k$ times a power product, 
possibly with negative exponents, of $x_{1}, \cdots, x_{n}$, and we can write $y_{1}+\cdots+y_{N}=\sum_{i=1}^{r} c_{i} u_{i}^{\prime} / u_{i}+v^{\prime}$, with each $c_{i}$ a constant of $k$ and $u_{1}, \cdots, u_{r}, v \in k(x)$. Assume, as we may, that each $u_{i}$ is an irreducible element of $k\left[x_{1}, \cdots, x_{n}\right]$ or is in $k$, and that $u_{i}$ divides $u_{j}$ only if $u_{i} \in k$. For any $f \in k\left[x_{1}, \cdots, x_{n}\right]$, the derivative $f^{\prime}$ is also in $k\left[x_{1}, \cdots, x_{n}\right]$, with the degree of $f^{\prime}$ in each $x_{i}$ at most that of $f$. In addition, if $f \in k\left[x_{1}, \cdots, x_{n}\right]$ is not a monomial then $f^{\prime}$ is not a multiple of $f$; for if $\left\{a_{i_{1} \cdots i_{n}}\right\} \subset k^{*},\left(i_{1}, \cdots, i_{n}\right)$ ranging over a finite subset of $Z^{n}$, then

$$
\begin{aligned}
& \sum\left(a_{i_{1} \cdots i_{n}} x_{1}^{i_{1}} \cdots x_{n}^{i_{n}}\right)^{\prime} \\
& \quad=\sum a_{i_{1} \cdots i_{n}} x_{1}^{i_{1}} \cdots x_{n}^{i_{n}}\left(\frac{a_{i_{1} \cdots i_{n}}^{\prime}}{a_{i_{1} \cdots i_{n}}}+i_{1} \frac{x_{1}^{\prime}}{x_{1}}+\cdots+i_{n} \frac{x_{n}^{\prime}}{x_{n}}\right)
\end{aligned}
$$

and if this were a multiple of $\sum a_{i_{1} \cdots i_{n}} x_{1}^{i_{1}} \cdots x_{n}^{i_{n}}$ then $a_{i_{1} \cdots i_{n}}^{\prime} / a_{i_{1} \cdots i_{n}}+$ $i_{1} x_{1}^{\prime} / x_{1}+\cdots+i_{n} x_{n}^{\prime} / x_{n}$ would be independent of $\left(i_{1}, \cdots, i_{n}\right)$, giving $\left(a_{i_{1} \cdots i_{n}} x_{1}^{i_{1}} \cdots x_{n}^{i_{n}} / a_{j_{1} \cdots j_{n}} x_{1}^{j_{1}} \cdots x_{n}^{j_{n}}\right)^{\prime}=0$ so that

$$
a_{i_{1} \cdots i_{n}} x_{1}^{i_{1}} \cdots x_{n}^{i_{n}} / a_{j_{1} \cdots j_{n}} x_{1}^{j_{1}} \cdots x_{n}^{j_{n}}
$$

is a constant, therefore an element of $k$, which is false for $\left(i_{1}, \cdots\right.$, $\left.i_{n}\right) \neq\left(j_{1}, \cdots, j_{n}\right)$. Therefore if $u_{i}$ is not a monomial the fraction $u_{i}^{\prime} / u_{i}$ is in lowest terms, with denominator $u_{i}$. Furthermore if $f \in k\left[x_{1}, \cdots, x_{n}\right]$ is an irreducible nonmonomial occurring as a factor of the denominator of $v$ exactly $s \geqq 1$ times, then $f$ occurs $(s+1)$ times in the denominator of $v^{\prime}$. Since each $y_{i}$ is a product of an element of $k$ by a power product of $x_{1}, \cdots, x_{n}$, comparing denominators in the equality $y_{1}+\cdots+y_{N}=\sum_{i=1}^{r} c_{i} u_{i}^{\prime} / u_{i}+v^{\prime}$ shows that each factor of the denominator of $v$ must be a monomial or in $k$, as must be each $u_{i}$ for which $c_{i} \neq 0$. Equating corresponding terms on the two sides of the equality gives the full theorem in the case where $x_{1}, \cdots, x_{n}$ are algebraically independent over $k$. Now consider the general case. Without loss of generality, as indicated at the end of the proof of Theorem 1 , we assume that $x_{1}, \cdots, x_{r}$ are algebraic over $k$ and that $x_{r+1}, \cdots, x_{n}$ are algebraically independent over $k$. Assume that $y_{1}, \cdots, y_{N}$ are as given and apply what we have already proved to the case where $k$ is replaced by its algebraic closure in $k\left(x_{1}, \cdots, x_{n}\right)$, that is by $k\left(x_{1}, \cdots, x_{r}\right)$. For each $i=1, \cdots, N$, we have to consider $\sum_{(i)} y_{j}$, with the sum extending over all $j=1, \cdots, N$ such that $y_{j} / y_{i} \in k\left(x_{1}, \cdots, x_{r}\right)$, and we obtain that each $\sum_{(i)} y_{j}$ is the derivative of an element in some elementary extension field of $k\left(x_{1}, \cdots, x_{n}\right)$ having the same constants, plus some further information. More precisely, we are reduced to the case where $y_{i} / y_{1} \in k\left(x_{1}, \cdots, x_{r}\right)$ for all $i=1, \cdots, N$, with the further knowledge that if $y_{1}$ is not algebraic over $k$ then $y_{1}+\cdots+y_{N}=\left(a y_{1}\right)^{\prime}$, for some $a \in k\left(x_{1}, \cdots, x_{r}\right)$, while if $y_{1}$ is algebraic over $k$ we can write 


$$
y_{1}+\cdots+y_{N}=\sum_{i=1}^{n} c_{i} x_{i}^{\prime} / x_{i}+\sum_{j} \gamma_{j} u_{j}^{\prime} / u_{j}+v^{\prime},
$$

where each $c_{i}$ and $\gamma_{j}$ is a constant of $k, j$ ranging over some finite set, and each $u_{j}$ and $v$ is an element of $k\left(x_{1}, \cdots, x_{r}\right)$. Since $x_{1}^{m}, \cdots, x_{r}^{m} \in k$ and $k$ contains a primitive $m^{\text {th }}$ root of unity, the field $k\left(x_{1}, \cdots, x_{r}\right)$ is a normal extension of $k$. We identity the galois groups $G=\operatorname{Aut}\left(k\left(x_{1}, \cdots, x_{r}\right) / k\right)$ and $\operatorname{Aut}\left(k\left(x_{1}, \cdots, x_{n}\right) / k\left(x_{r+1}, \cdots, x_{n}\right)\right)$. For any $y \in k\left(x_{1}, \cdots, x_{n}\right)$ that is exponential over $k$ we have $y^{m} \in$ $k\left(x_{r+1}, \cdots, x_{n}\right)$, so that for each $\sigma \in G$ we have $\sigma y / y$ an $m^{\text {th }}$ root of unity. We claim that not only $y_{1}+\cdots+y_{N}$, but also each $y_{i}$, is of the form indicated above. To prove this, suppose the contrary, so that $N>1$, and suppose that $N$ is minimal for a counterexample. Since each $\sigma \in G$ is a differential automorphism of $k\left(x_{1}, \cdots, x_{n}\right)$, $\sigma\left(y_{1}+\cdots+y_{N}\right)$ is also of the desired form, hence also

$$
\sigma\left(y_{1}+\cdots+y_{N}\right)-\left(\sigma y_{1} / y_{1}\right)\left(y_{1}+\cdots+y_{N}\right),
$$

which is a sum of constant multiples of $y_{2}, \cdots, y_{N}$. Since $y_{2} / y_{1} \notin k$ we can choose $\sigma$ such that $\sigma\left(y_{2} / y_{1}\right) \neq y_{2} / y_{1}$, and then the minimality of $N$ implies that $y_{2}$ is of the desired form, hence each of $y_{1}, \cdots, y_{N}$. That is, we can write each $y_{i}$ in the form indicated for $y_{1}+\cdots+y_{N}$. We are therefore done in the case where $y_{i}$ is algebraic over $k$ and reduced to the statement that $y_{i}=\left(a y_{i}\right)^{\prime}$ for some $a \in k\left(x_{1}, \cdots, x_{r}\right)$ if $y_{i}$ is not algebraic over $k$. Here $a$ is unique, since if we also have $a_{1} \in k\left(x_{1}, \cdots, x_{r}\right)$ and $y_{i}=\left(a_{1} y_{i}\right)^{\prime}$, then $\left(\left(a-a_{1}\right) y_{i}\right)^{\prime}=0$, so $\left(a-a_{1}\right) y_{i}$ is constant, hence in $k$, contradicting the transcendence of $y_{i}$ over $k$ unless $a=a_{1}$. For any $\sigma \in G$ we have $\sigma y_{i}=\left((\sigma a)\left(\sigma y_{i}\right)\right)^{\prime}$, so that $y_{i}=\left((\sigma a) y_{i}\right)^{\prime}$, so that $\sigma a=a$. Thus we have $a \in k$, which was the only item remaining to be shown.

CoRollary. If $f_{1}, \cdots, f_{n}, g_{1}, \cdots, g_{n}$ are algebraic functions of a complex variable and no two of $g_{1}, \cdots, g_{n}$ differ by a constant, then $f_{1} e^{g_{1}}+\cdots+f_{n} e^{g}$ is the derivative of an elementary function if and only if each $f_{i} e^{g_{i}}$ is.

We recall that an elementary function of a complex variable is an element of an elementary differential extension field of the field of rational functions. The Corollary, a well-known result of Liouville [5, p. 49], follows immediately from the fact that the exponential of a nonconstant algebraic function is not an algebraic function.

Theorem 2 and its proof generalize immediately to the case of differential fields with more than one given derivation. We merely indicate the changes necessary in its statement: We drop the word "ordinary". Instead of being given $y_{1}, \cdots, y_{N} \in k(x)$, we are now 
given $N$ nonzero functions $y_{1}, \cdots, y_{N}$ from the set of given derivations into $k(x)$ such that for each $i=1, \cdots, N$ and each given derivation $D, y_{i}(D)$ is either exponential over $k$ or zero, and such that if $i, j=1, \cdots, N$ and $D$ and $\delta$ are given derivations such that $y_{i}(D) \neq 0$ and $y_{j}(\delta) \neq 0$, then $y_{i}(D) / y_{j}(\delta)$ is in $k$ if and only if $i=j$. To say that a function $y$ on the set of given derivations of $k(x)$ is the derivative of an element $z$ of some differential extension field will of course signify that $y(D)=D z$ for each given derivation $D$. Finally, the statement that $y_{i}$ is or is not algebraic over $k$ is to be interpreted as meaning that $y_{i}(D)$ is or is not algebraic over $k$ for any given derivation $D$ for which $y_{i}(D) \neq 0$.

In the last part of the theorem it is stated that under certain conditions $y_{i}$ is the derivative of $a y_{i}$, for some $a \in k$. One of the conditions is that $y_{i}$ is not algebraic over $k$. That this condition is necessary is seen by the example $k=\boldsymbol{R}(\tan x)$, with $\boldsymbol{R}$ the real numbers and $(\tan x)^{\prime}=\tan ^{2} x+1$, and $y=\sec x$ : here $y$, which is both exponential and algebraic over $k$, is the derivative of $\log (\sec x+$ $\tan x$ ), but not of any multiple of itself by an element of $k$. However if the element $y$, which is exponential and algebraic over $k$, is the derivative of an element of $k(y)$, then it is the derivative of $a y$, for some $a \in k$ : for if $m=[k(y): k]$, then each element of $k(y)$ can be uniquely written in the form $\sum_{i=0}^{m-1} a_{i} y^{i}$, with each $a_{i} \in k$, and from the equation $y=\left(\sum a_{i} y^{i}\right)^{\prime}$ we deduce $y=\left(a_{1} y\right)^{\prime}$ by homogeneity, since each $a_{i} y^{i}$ is exponential over $k$. This comment will apply to the case of an algebraic function $y$ of the complex variable $x$ some power of which is in the rational function field $C(x)$ if $\int y d x$ is an elementary function of $x$ and the differential $f d x$ has no residues, for here we can write $y=\sum c_{i} u_{i}^{\prime} / u_{i}+v^{\prime}$, with each $c_{\imath} \in \boldsymbol{C}$ and each $u_{i}, v \in \boldsymbol{C}(x, y)$, and if we arrange, as we can, that $\left\{c_{2}\right\}$ are linearly independent over the rational numbers $\boldsymbol{Q}$, then the absence of residues of $y d x=$ $\sum c_{i} d u_{i} / u_{i}+d v$ implies that each $u_{i} \in \boldsymbol{C}$, so that $y=v^{\prime}$, with $v \in \boldsymbol{C}(x, y)$.

The statement of Theorem 2 fails without the condition that $k$ contain a primitive $m^{\text {th }}$ root of unity. For a counterexample, consider the differential field of functions on the positive real line $k=\boldsymbol{R}(x)$, where $x^{\prime}=1$, and its differential extension field $\boldsymbol{R}(t)$, where $t=x^{1 / m}$, with $m \in \boldsymbol{Z}, m>2$, which has the same subfield of constants $\boldsymbol{R}$ and in which $t^{\prime}=t / m x$. The element $(1-t)^{\prime} /(1-t)$ is the derivative of an element in an elementary extension field of $\boldsymbol{R}(t)$ having the same constants. However

$$
\frac{(1-t)^{\prime}}{1-t}=\frac{-t^{\prime}}{1-t}=\frac{-t^{\prime}\left(1+t+\cdots+t^{m-1}\right)}{1-t^{m}}=-\frac{t+t^{2}+\cdots+t^{m}}{m x(1-x)}
$$

and we claim that $t / m x(1-x)$ cannot be written in the form 
$\sum_{i=1}^{n} c_{i} u_{i}^{\prime} / u_{i}+v^{\prime}$ with $c_{1}, \cdots, c_{n} \in R$ and $u_{1}, \cdots, u_{n}, v \in \boldsymbol{R}(t)$. For

$$
\sum_{i=1}^{n}\left(c_{i} u_{i}^{\prime} / u_{i}+v^{\prime}\right) d x=\sum_{i=1}^{n} c_{i} d u_{i} / u_{i}+d v
$$

has a real residue at the place $t=\zeta$, where $\zeta$ is a primitive $m^{\text {th }}$ root of unity, while the residue there of $t d x / m x(1-x)$ is $-\zeta / m \notin \boldsymbol{R}$.

3. In this section we consider the differential field $C(x)$ of rational functions of a complex variable $x$, with $x^{\prime}=1$, and functions that are exponential over $C(x)$, that is elements $y$ of a differential extension field of $C(x)$ having the same constants $C$ and such that $y^{\prime} / y=w \in C(x)$. Since $\int w(x) d x$ is an elementary function, that is an element of an elementary differential extension field of $C(x)$ with the same constants, $y=\exp \int w(x) d x$ is also an elementary function. The element $y$ will be in $C(x)$ if and only if $w$ is a finite sum of elements of the form (integer) $/(x-($ element of $C)$ ); $y$ will be an algebraic function, that is algebraic over $C(x)$, if and only if some power of $y$ is in $\boldsymbol{C}(x)$, and a necessary and sufficient condition for this is that $w$ be a finite sum of elements of the form (rational number) $/(x-$ (element of $C)$ ).

We ask when $\int y d x$ is an elementary function. If $y$ is transcendental over $C(x)$, a condition for this is given in Theorem 2, and the next to the last paragraph of $\S 2$ gives a partial extension of this condition to the case where $y$ is algebraic over $C(x)$ : If $y$ is transcendental over $C(x)$ or if $y$ is algebraic over $C(x)$ and the differential $y d x$ has no residues, then $\int y d x$ is elementary if and only if there exists $a \in C(x)$ such that $y=(a y)^{\prime}$ or, equivalently, $1=a^{\prime}+a w$. If $y \notin \boldsymbol{C}(x)$ and such an $a$ exists, it is unique, for if also $a_{1} \in \boldsymbol{C}(x)$ and $1=a_{1}^{\prime}+a_{1} w$, then $\left(a-a_{1}\right)^{\prime}+\left(a-a_{1}\right) w=0$, so $\left(\left(a-a_{1}\right) y\right)^{\prime}=0$, so $\left(a-a_{1}\right) y \in C$, which is possible only if $a=a_{1}$.

For a given $w \in C(x)$ we can find $a \in C(x)$ such that $a^{\prime}+a w=1$, if such an $a$ exists, by examining the partial fraction expansions of $a$ and $w$. We get immediately that $a$ can have a pole at a finite place $x=\alpha$, where $\alpha \in C$, only if $x=\alpha$ is a pole of $w$, and that if $a$ has a pole of order $r \geqq 1$ at $x=\alpha$ then the principal part of $w$ at $x=\alpha$ must be $r /(x-\alpha)$, that is $w-r /(x-\alpha)$ must be finite at $x=\alpha$. Similarly, if $a$ has a pole of order $r>1$ at $x=\infty$, the expansion of $w$ at $x=\infty$ must start with $-r / x$, that is $x w+r$ must vanish at $x=\infty$. If $a$ has a pole of order 1 at $x=\infty$, then $w$ must vanish there. Thus for any given $w$ the poles of $a$, if any, must occur among the poles of $w$ and the place $x=\infty$, and to at most certain specified orders at these places. Thus the partial fraction 
expansion of $a$ can be written out with indeterminate coefficients in $C$ and finding $a \in C(x)$ such that $a^{\prime}+a w=1$ reduces to solving a system of linear equations in the coefficients of the expansion of $a$. Note that if $w$ has a pole of order $r \geqq 1$ at any place of $C(x)$ over $C$, finite or infinite, and $a$ is finite at this place, then $a$ must have a zero there of order at least $r$.

Michael Tarter, in connection with his work on approximating inverse cumulative distribution functions [8], [9] has asked whether certain natural cumulative distribution functions, or their inverse functions, are elementary. The cumulative distribution function $F(x)$ corresponding to the probability distribution $y(x)$ is given by $F(x)=$ $\int^{x} y(t) d t$, so that $F^{\prime}=y$, and his case of special interest is that in which $y^{\prime} / y=w \in \boldsymbol{C}(x)$, in particular the case of Pearson distributions [1, pp. 148-154], where $w(x)=\left(x-c_{0}\right) /\left(c_{1}+c_{2} x+c_{3} x^{2}\right)$, with each $c_{i}$ constant. Here $y$ is an elementary function, and in virtue of the theorem of Ritt to the effect that if the inverse function of the integral of an elementary function is elementary then so is the integral (see [5, p. 87] or, for a modern exposition, [7]), $F^{-1}$ can be elementary only if $F$ is elementary.

If we have a Pearson distribution $y \notin C(x)$, then $w=y^{\prime} / y$ must be of one of the following forms:

(1) $w=p(x-b), p, b \in \boldsymbol{C}, p \neq 0$

(2) $w=p+q /(x-b), p, q, b \in C, p \neq 0$

(3) $w=p /\left(x-b_{1}\right)+q /\left(x-b_{2}\right), p, q, b_{1}, b_{2} \in \boldsymbol{C}, b_{1} \neq b_{2}, p \notin \boldsymbol{Z}$

(4) $w=p /(x-b)+q /(x-b)^{2}, p, q, b \in \boldsymbol{C}, q \neq 0$.

In each of these cases, $y$ is transcendental over $C(x)$ except in case (3) with $p, q \in \boldsymbol{Q}$. Special cases of (1), (2), (3) respectively that are of statistical interest are $w=-2 x$, where $y=e^{-x^{2}}$ (normal distribution), $w=-1+(\lambda-1) / x$, with $\lambda \in \boldsymbol{Q}$, where $y=x^{\lambda-1} e^{-x}$ (Gamma distribution), and $w=(p-1) / x-(q-1) /(1-x)$, with $p, q \in \boldsymbol{Q}$, where $y=x^{p-1}(1-x)^{q-1}$ (Beta distribution, whose cumulative distribution function is the Chebyshev integral $\int x^{p-1}(1-x)^{q-1} d x$, cf. [5, p. 37]). To see whether or not $\int y d x$ is elementary in the various cases (1), (2), (3), (4), we must check whether there exists $a \in C(x)$ such that $a^{\prime}+a w=1$. Note that $a$ can have poles only at $x=b, x=b_{1}, x=b_{2}$ and $x=\infty$, and of orders depending on $p$ and $q$. In case (1), $a$ can have no poles, therefore we must have $a \in C$, and for no such $a$ can we have $a^{\prime}+a w=1$, so $\int y d x$ is nonelementary. In case (2) $a$ must be finite at $x=\infty$ and can have poles at $x=b$ only if $q \in Z$, $q>0$. We deduce that $\int y d x$ is nonelementary unless $q \in Z, q \geqq 0$, in which case we know from elementary calculus that $\int y d x=\int(x-$ 
$b)^{q} e^{p x} d x$ is elementary. In case (3) we have $\int y d x=\int\left(x-b_{1}\right)^{p}\left(x-b_{2}\right)^{q} d x$, and integration by parts shows that computing the latter integral is equivalent to computing either

$$
\int\left(x-b_{1}\right)^{p+1}\left(x-b_{2}\right)^{q-1} d x \text { or } \int\left(x-b_{1}\right)^{p-1}\left(x-b_{2}\right)^{q+1} d x \text {. }
$$

Using this fact repeatedly, we get $\int y d x$ elementary if $q \in Z$. The substitution $x_{1}=1 /\left(x-b_{1}\right)$ shows that $\int y d x$ is elementary if $p+$ $q \in \boldsymbol{Z}$. Hence, slightly more generally than case (3), if $w=p /\left(x-b_{1}\right)+$ $q /\left(x-b_{2}\right)$, with $p, q, b_{1}, b_{2} \in C$ and $b_{1} \neq b_{2}$, we have $\int y d x$ elementary if either $p$, or $q$, or $p+q$ is in $\boldsymbol{Z}$. We claim that, conversely, $\int y d x$ is nonelementary if none of $p, q$, or $p+q$ is in $Z$. First, if $y$ is algebraic over $\boldsymbol{C}(x)$ then $p, q \in \boldsymbol{Q}$ and an easy computation shows that $y d x=\left(x-b_{1}\right)^{p}\left(x-b_{2}\right)^{q} d x$ has no residues. Therefore we need only check the existence of $a \in C(x)$ such that $a^{\prime}+a w=1$. We see that $a$ can have no poles when $x$ is finite and a pole of order at most one at $x=\infty$, so that $a=\alpha x+\beta$, with $\alpha, \beta \in C$, and this $a$ does not work; this proves our contention. In case (4), we claim that $\int y d x$ is never elementary. For here $\int y d x=\int(x-b)^{p} e^{-q /(x-b)} d x$, and integration by parts shows that if $p \neq-1$ then the computation of $\int y d x$ is equivalent to that of $\int(x-b)^{p-1} e^{-q /(x-b)} d x$, so that we may suppose $p \geqq-1$. Then $a$, which can have no poles for finite $x$, can have a pole of order at most one at $x=\infty$, so again $a=\alpha x+\beta$, with $\alpha, \beta \in C$, and again this $a$ fails to satisfy $a^{\prime}+a w=1$.

Tarter has also asked whether for a distribution function of the form $c_{1} y_{1}+\cdots+c_{n} y_{n}$, where each $c_{i} \in \boldsymbol{C}$ and each $y_{i}^{\prime} / y_{i}=w_{i} \in \boldsymbol{C}(x)$, the inverse of the cumulative distribution function $F(x)=\int\left(c_{1} y_{1}+\right.$ $\left.\cdots+c_{n} y_{n}\right) d x$ can be elementary. By the result of Ritt already referred to, this can happen only if $F(x)$ itself is elementary, which will be true if each $\int y_{2} d x$ is elementary. By Theorem 2, if $F(x)$ is elementary, then for each $i=1, \cdots, n, \int\left(\sum_{(j)} c_{j} y_{j}\right) d x$ is elementary, where $j$ ranges over the indices $1,2, \cdots, n$ for which $y_{j} / y_{i} \in \boldsymbol{C}(x)$, which is equivalent to $w_{j}-w_{i}$ being a finite sum of elements of the form (integer) $/\left(x-(\right.$ element of $C)$ ). In particular, if each $c_{i} \neq 0$, then for each $i=1, \cdots, n$ there must be a $j=1, \cdots, n$ such that $j \neq i$ and $w_{j}-w_{i}$ is of the indicated special form, a rare circumstance. In any case the problem of finding when for given $y_{1}, \cdots, y_{n}$ of the above type there exist constants $c_{1}, \cdots, c_{n}$ not all zero such that $c_{1} y_{1}+\cdots+c_{n} y_{n}$ has an elementary integral reduces to the special case where each $y_{i}$ is of the form $y_{i}=f_{i} y$, where $y^{\prime} / y=w(x) \in C(x)$ 
and $f_{i} \in C(x)$ is such that $\int f_{i} y d x$ is nonelementary; we therefore have to find for which $c_{1}, \cdots, c_{n} \in C$ there exists a function $A \in C(x)$ such that $c_{1} y_{1}+\cdots+c_{n} y_{n}=(A y)^{\prime}$, or equivalently $c_{1} f_{1}+\cdots+c_{n} f_{n}=$ $A^{\prime}+A w$, a generalization of the type of equation handled before. One case of special interest is that in which we are given $y$ such that $y^{\prime} / y=w \in C(x)$ and take each $y_{i}$ to be of the form $y_{i}(x)=$ $y\left(\alpha_{i} x+\beta_{i}\right)$, where $\left(\alpha_{1}, \beta_{1}\right), \cdots,\left(\alpha_{n}, \beta_{n}\right)$ are distinct elements of $C^{2}$ and no $\alpha_{i}$ is zero. In this case $w_{i}=y_{i}^{\prime} / y_{i}=\alpha_{i} w\left(\alpha_{i} x+\beta_{i}\right)$. It is easy to verify that for no such $\left(\alpha_{1}, \beta_{1}\right), \cdots,\left(\alpha_{n}, \beta_{n}\right)$ can there exist constants $c_{1}, \cdots, c_{n}$ not all zero such that $\int\left(c_{1} y_{1}+\cdots+c_{n} y_{n}\right) d x$ is elementary in the cases where $w$ is of Pearson type (2) with $q \notin Z$, or of type (3) with $p, q, p+q \notin Z$ and $p \neq q$, or of type (4). But we can find such $\left(\alpha_{1}, \beta_{1}\right), \cdots,\left(\alpha_{n}, \beta_{n}\right), c_{1}, \cdots, c_{n}$ for Pearson type (1), say for the normal distribution $y=e^{-x^{2}}, w=-2 x$, where $y(x)-y(-x)=0$ has an elementary integral.

\section{REFERENCES}

1. M. G. Kendall and A. Stuart, The Advanced Theory of Statistics, vol. 1, Hafner, New York, 1963.

2. E. Kolchin, Algebraic groups and algebraic dependence, Amer. J. Math., 90 (1968), 1151-1164.

3. - Differential Algebra and Algebraic Groups, Academic Press, New York, 1973.

4. R. Risch, The problem of integration in finite terms, Trans. Amer. Math. Soc., 139 (1969), 167-189.

5. J. F. Ritt, Integration in Finite Terms, Columbia Univ. Press, New York, 1948.

6. M. Rosenlicht, On Liouville's theory of elementary functions, to appear.

7. M. Singer, Functions Satisfying Elementary Relations, Berkeley dissertation, 1974.

8. M. Tarter, Inverse cumulative approximation and applications, Biometrika, 55 (1968), 29-41.

9. - Estimation procedures for simulation applications, in Perspectives in Biometrics, vol. 1, ed. by R. Elashoff, Academic Press, New York, 1974.

Received October 23, 1974. Research supported by National Science Foundation grant number GP-37492X.

University of CALifornia, Berkeley 


\title{
PACIFIC JOURNAL OF MATHEMATICS
}

\author{
EDITORS
}

RICHARD ARENS (Managing Editor)

University of California

Los Angeles, California 90024

R. A. Beaumont

University of Washington

Seattle, Washington 98105
J. DugundJI

Department of Mathematics University of Southern California Los Angeles, California 90007

D. Gilbarg and J. Milgram

Stanford University

Stanford, California 94305

\section{ASSOCIATE EDITORS}

E. F. BECKENBACH

B. H. NeUManN

F. WOLF

K. YosHIDA

\section{SUPPORTING INSTITUTIONS}

UNIVERSITY OF BRITISH COLUMBIA

GALIFORNIA INSTITUTE OF TECHNOLOGY

UNIVERSITY OF CALIFORNIA

MONTANA STATE UNIVERSITY

UNIVERSITY OF NEVADA

NEW MEXICO STATE UNIVERSITY

OREGON STATE UNIVERSITY

UNIVERSITY OF OREGON

OSAKA UNIVERSITY
UNIVERSITY OF SOUTHERN CALIFORNIA

STANFORD UNIVERSITY

UNIVERSITY OF TOKYO

UNIVERSITY OF UTAH

WASHINGTON STATE UNIVERSITY

UNIVERSITY OF WASHINGTON

AMERICAN MATHEMATICAL SOCIETY NAVAL WEAPONS CENTER 


\section{Pacific Journal of Mathematics}

\section{Vol. 57, No. $1 \quad$ January, 1975}

Keith Roy Allen, Dendritic compactification ......................... 1

Daniel D. Anderson, The Krull intersection theorem ..................... 11

George Phillip Barker and David Hilding Carlson, Cones of diagonally dominant

matrices .............................................

David Wilmot Barnette, Generalized combinatorial cells and facet splitting ........

Stefan Bergman, Bounds for distortion in pseudoconformal mappings ............

Nguyên Phuong Các, On bounded solutions of a strongly nonlinear elliptic

equation ...............................................

Philip Throop Church and James Timourian, Maps with 0-dimensional critical

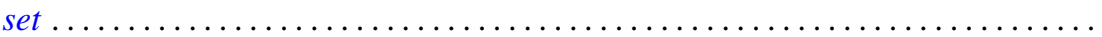

G. Coquet and J. C. Dupin, Sur les convexes ubiquitaires ................. 67

Kandiah Dayanithy, On perturbation of differential operators............... 85

Thomas P. Dence, A Lebesgue decomposition for vector valued additive set

functions .............................................. 91

John Riley Durbin, On locally compact wreath products.................. 99

Allan L. Edelson, The converse to a theorem of Conner and Floyd ............. 109

William Alan Feldman and James Franklin Porter, Compact convergence and the

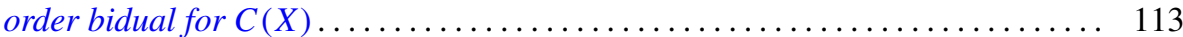

Ralph S. Freese, Ideal lattices of lattices......................... 125

R. Gow, Groups whose irreducible character degrees are ordered by divisibility ... 135

David G. Green, The lattice of congruences on an inverse semigroup ............ 141

John William Green, Completion and semicompletion of Moore spaces .......... 153

David James Hallenbeck, Convex hulls and extreme points of families of starlike and close-to-convex mappings .................................. 167

Israel (Yitzchak) Nathan Herstein, On a theorem of Brauer-Cartan-Hua type ...... 177

Virgil Dwight House, Jr., Countable products of generalized countably compact spaces ............................................ 183

John Sollion Hsia, Spinor norms of local integral rotations. I . . . . . . ......... 199

Hugo Junghenn, Almost periodic compactifications of transformation

semigroups....................................

Shin'ichi Kinoshita, On elementary ideals of projective planes in the 4-sphere and oriented $\Theta$-curves in the 3 -sphere ............................ 217

Ronald Fred Levy, Showering spaces............................ 223

Geoffrey Mason, Two theorems on groups of characteristic 2-type............. 233

Cyril Nasim, An inversion formula for Hankel transform ................... 255

W. P. Novinger, Real parts of uniform algebras on the circle ................ 259

T. Parthasarathy and T. E. S. Raghavan, Equilibria of continuous two-person

games............................................... 265

John Pfaltzgraff and Ted Joe Suffridge, Close-to-starlike holomorphic functions of several variables ....................................... 271

Esther Portnoy, Developable surfaces in hyperbolic space .................. 281

Maxwell Alexander Rosenlicht, Differential extension fields of exponential type . . . 289

Keith William Schrader and James Lewis Thornburg, Sufficient conditions for the

existence of convergent subsequences ........................... 301

Joseph M. Weinstein, Reconstructing colored graphs .................. 307 